NEIP-03-004

hep-th/0309147

\title{
Supersymmetric quantum mechanics from wrapped branes
}

\author{
Rafael Hernández ${ }^{1}$ and Konstadinos Sfetsos ${ }^{2}$ \\ ${ }^{1}$ Institut de Physique, Université de Neuchâtel \\ Breguet 1, CH-2000 Neuchâtel, Switzerland \\ rafael.hernandez@unine.ch \\ 2 Department of Engineering Sciences, University of Patras \\ 26110 Patras, Greece \\ sfetsos@des.upatras.gr
}

\begin{abstract}
We explicitly construct a solution of eight-dimensional gauged supergravity representing D6-branes wrapped on six-cycles inside Calabi-Yau fourfolds. The solution preserves two supercharges and asymptotically is a cone with the coset space $S U(2)^{4} / U(1)^{3}$ as its base. It is shown to correspond to an M-theory compactification on a Calabi-Yau manifold with $S U(5)$ holonomy and we discuss in detail its geometrical and topological features. We also construct a family of related higher dimensional metrics having $S U(n+1)$ holonomy, which of course have no brane interpretation.
\end{abstract}




\section{Introduction}

D6-branes have a purely geometrical origin in eleven dimensions as the Kaluza-Klein monopole. When the amount of supersymmetry on their worldvolume is reduced by wrapping them on supersymmetric cycles they admit an eleven-dimensional description in terms of compactifications of M-theory on manifolds with reduced holonomy [1]. Keeping some unbroken supersymmetry as the brane wraps a cycle requires coupling the theory to an external R-current, which is then related to the spin connection on the cycle. The resulting theory is a topologically twisted field theory [2]. At a more technical level, gauged supergravities have provided the adequate arena to perform the twist relating the gauge and spin connections [3] (see for instance [4] for a review).

In this way compactifications of M-theory on manifolds with reduced holonomy arise as the local eleven-dimensional version of backgrounds in eight-dimensional gauged supergravity describing D6-branes wrapped on diverse supersymmetric cycles. M-theory on Calabi-Yau threefolds corresponds to D6-branes wrapped on supersymmetric two-cycles inside twofolds [5, 6], compactification on Calabi-Yau fourfolds comes from D6-branes wrapped on four-cycles inside threefolds [7], compactification on manifolds with $G_{2}$ holonomy arises as the eleven-dimensional description of D6-branes wrapped on supersymmetric three-cycles inside Calabi-Yau threefolds [5, 8, 9], and M-theory on eight-manifolds with Spin(7) holonomy corresponds to D6-branes wrapped on four-cycles inside manifolds with $G_{2}$ holonomy [10]. The deformation of this purely geometrical solutions by non-trivial background fluxes has also been extensively studied [11]-[15] (a more complete list of references on branes of different dimension wrapped on various supersymmetric cycles can be found in [16]).

In this letter we will consider the case of D6-branes wrapped on six-cycles inside CalabiYau fourfolds. The remaining theory on the worldvolume of the branes will be a supersymmetric quantum mechanics with two supercharges, and when lifted to eleven dimensions the solution will correspond to M-theory compactified on a Calabi-Yau manifold of $S U(5)$ holonomy. In section 2 we will present the most general supersymmetry preserving solution and its deformation by a non-trivial background flux. In section 3 we generalize our results to provide a family of metrics having $S U(n+1)$ holonomy and conclude with some remarks. 


\section{D6-branes wrapped on supersymmetric six-cycles}

Before constructing our solution let us briefly review the relevant sector of gauged supergravity in eight dimensions which was originally constructed by Salam and Sezgin [17] through a Scherk-Schwarz compactification of eleven-dimensional supergravity [18] on an $S U(2)$ group manifold. The field content of the theory consists of the metric $g_{\mu \nu}$, a dilaton $\Phi$, five scalars given by a unimodular $3 \times 3$ matrix $L_{\alpha}^{i}$ in the coset $S L(3, \mathbb{R}) / S O(3)$ and an $S U(2)$ gauge potential $A_{\mu}$, all in the gravity sector, and a three-form and three vector fields coming from reduction of the eleven-dimensional three-form. ${ }^{1}$ In addition, on the fermion side we have the pseudo-Majorana spinor $\psi_{\mu}$ and the gaugino $\chi_{i}$.

The supersymmetry variations for the gaugino and the gravitino are given by

$$
\begin{aligned}
\delta \chi_{i} & =\frac{1}{2}\left(P_{\mu i j}+\frac{2}{3} \delta_{i j} \partial_{\mu} \Phi\right) \hat{\Gamma}^{j} \Gamma^{\mu} \epsilon-\frac{1}{4} e^{\Phi} F_{\mu \nu i} \Gamma^{\mu \nu} \epsilon-\frac{g}{8} e^{-\Phi}\left(T_{i j}-\frac{1}{2} \delta_{i j} T\right) \epsilon^{j k l} \hat{\Gamma}_{k l} \epsilon \\
& -\frac{1}{144} e^{\Phi} G_{\mu \nu \rho \sigma} \hat{\Gamma}_{i} \Gamma^{\mu \nu \rho \sigma} \epsilon-\frac{1}{24} e^{-\Phi} \epsilon^{k l n} G_{\mu \nu n}\left(\hat{\Gamma}_{k l i}+4 \hat{\Gamma}_{k} \delta_{l i}\right) \Gamma^{\mu \nu} \epsilon=0 \\
\delta \psi_{\lambda} & =\mathcal{D}_{\lambda} \epsilon+\frac{1}{24} e^{\Phi} F_{\mu \nu}^{i} \hat{\Gamma}_{i}\left(\Gamma_{\lambda}^{\mu \nu}-10 \delta_{\lambda}^{\mu} \Gamma^{\nu}\right) \epsilon-\frac{g}{288} e^{-\Phi} \epsilon_{i j k} \hat{\Gamma}^{i j k} \Gamma_{\lambda} T \epsilon \\
& -\frac{1}{96} e^{\Phi} G_{\mu \nu \rho \sigma}\left(\Gamma_{\lambda}^{\mu \nu \rho \sigma}-4 \delta_{\lambda}^{\mu} \Gamma^{\nu \rho \sigma}\right) \epsilon-\frac{1}{48} e^{-\Phi} \epsilon^{i j k} G_{\mu \nu k} \hat{\Gamma}_{i j}\left(\Gamma_{\lambda}{ }^{\mu \nu}-10 \delta_{\lambda}^{\mu} \Gamma^{\nu}\right) \epsilon=0
\end{aligned}
$$

where the Yang-Mills field strength is $F_{\mu \nu}^{\alpha}$, the covariant derivative is defined as

$$
\mathcal{D}_{\lambda} \epsilon=\partial_{\lambda} \epsilon+\frac{1}{4} \omega_{\lambda}^{a b} \Gamma_{a b} \epsilon+\frac{1}{4} Q_{\lambda i j} \hat{\Gamma}^{i j} \epsilon
$$

where $P_{\gamma i j}$ and $Q_{\gamma i j}$ are, respectively, the symmetric and antisymmetric quantities entering the Cartan decomposition of the $S L(3, \mathbb{R}) / S O(3)$ coset, defined through

$$
P_{\mu i j}+Q_{\mu i j} \equiv L_{i}^{\alpha}\left(\partial_{\mu} \delta_{\alpha}^{\beta}-g \epsilon_{\alpha \beta \delta} A_{\mu}^{\delta}\right) L_{\beta j}
$$

and $T_{i j}$ is the $T$-tensor defining the potential energy associated to the scalar fields,

$$
T^{i j} \equiv L_{\alpha}^{i} L_{\beta}^{j} \delta^{\alpha \beta}
$$

with $T \equiv T_{i j} \delta^{i j}$, while $L_{\alpha}^{i}$ satisfy $L_{\alpha}^{i} L_{j}^{\alpha}=\delta_{j}^{i}, L_{\alpha}^{i} L_{\beta}^{j} \delta_{i j}=g_{\alpha \beta}, L_{\alpha}^{i} L_{\beta}^{j} g^{\alpha \beta}=\delta^{i j}$. As usual, curved directions are labeled by greek indices, while flat ones are labeled by latin, and $\mu, a=0,1, \ldots, 7$ are spacetime coordinates, while $\alpha, i=8,9,10$ are in the group manifold.

\footnotetext{
${ }^{1}$ Reduction of the eleven-dimensional three-form also produces a scalar and three two-forms. However, we will set all these fields to zero.
} 
Note also that upper indices in the gauge field, $A_{\mu}^{\alpha}$, are always curved, and that the field strengths in eight dimensional curved space are defined as

$$
G_{\mu \nu \rho \sigma}=e^{-4 \Phi / 3} e_{\mu}^{a} e_{\nu}^{b} e_{\rho}^{c} e_{\sigma}^{d} F_{a b c d}, \quad G_{\mu \nu \alpha \beta}=e^{2 \Phi / 3} e_{\mu}^{a} e_{\nu}^{b} L_{\alpha}^{i} L_{\beta}^{j} F_{a b i j}=\epsilon_{\alpha \beta \gamma} G_{\mu \nu \gamma},
$$

with $G_{\mu \nu \alpha \beta}$ generated by three vector fields $B_{\mu \alpha}$ as

$$
G_{\mu \nu \alpha}=\partial_{\mu} B_{\nu \alpha}-\partial_{\nu} B_{\mu \alpha}
$$

We should point out that the above definition ignores the contribution to the $S U(2)$ gauge field $A_{\mu}^{\alpha}$ which is of the form $\epsilon_{\alpha \beta}^{\gamma} A_{\mu}^{\beta} B_{\nu \gamma}-(\mu \leftrightarrow \nu)$. This will be justified by the form of our ansatz which will make this term to vanish identically. These vector fields generate also a contribution to the three three-form field strength $G_{\mu \nu \rho \alpha}$ of the form $\epsilon_{\alpha \beta \gamma} F_{\mu \nu}^{\beta} B_{\rho \gamma}$. Again the absence of these terms will be justified by the form of our ansatz.

Let us now introduce the system under study. We will consider a D2-D6 brane system, with the D6-branes wrapped on supersymmetric six-cycles inside Calabi-Yau fourfolds, that is, divisors. As a starting point, we will take the six-cycle to be a direct product of three two-spheres of different radii, $S^{2} \times \bar{S}^{2} \times \tilde{S}^{2}$ (in an obvious notation). The deformation on the worl-dvolume of the D6-branes will then be described by a metric of the form

$$
d s_{8}^{2}=-e^{2 f} d t^{2}+d \rho^{2}+\alpha_{1}^{2} d \Omega_{2}^{2}+\alpha_{2}^{2} d \bar{\Omega}_{2}^{2}+\alpha_{3}^{2} d \tilde{\Omega}_{2}^{2},
$$

with the line elements for the spheres (normalized to have scalar curvature equal to 2)

$$
d \Omega_{2}^{2}=d \theta^{2}+\sin ^{2} \theta d \phi^{2}, \quad d \bar{\Omega}_{2}^{2}=d \bar{\theta}^{2}+\sin ^{2} \bar{\theta} d \bar{\phi}^{2}, \quad d \tilde{\Omega}_{2}^{2}=d \tilde{\theta}^{2}+\sin ^{2} \tilde{\theta} d \tilde{\phi}^{2}
$$

and $f, \alpha_{1}, \alpha_{2}$ and $\alpha_{3}$ depend only on the radial variable $\rho$. The same dependence will also hold for all additional fields that we will turn on. It will be useful to introduce a triplet of Maurer-Cartan 1-forms on $S^{2}$,

$$
\sigma_{1}=\sin \theta d \phi, \quad \sigma_{2}=d \theta, \quad \sigma_{3}=\cos \theta d \phi
$$

that obey $d \sigma_{i}=\frac{1}{2} \epsilon_{i j k} \sigma_{j} \wedge \sigma_{j}$, so that they resemble the triplet of Maurer-Cartan forms on $S^{3}$, although obviously only two of them are the independent ones. Similar triplets, $\bar{\sigma}_{i}$ and $\tilde{\sigma}_{i}$, can also be defined on the remaining spheres, $\bar{S}^{2}$ and $\tilde{S}^{2}$. In the natural frame

$$
e^{0}=e^{f} d t, \quad e^{7}=d \rho, \quad e^{i}=\alpha_{1} \sigma^{i}, \quad \bar{e}^{i}=\alpha_{2} \bar{\sigma}^{i}, \quad \tilde{e}^{i}=\alpha_{3} \tilde{\sigma}^{i}, \quad i=1,2,
$$


the expressions for the spin connection for the metric (2.7) are

$$
\begin{aligned}
& \omega^{07}=\frac{d f}{d \rho} e^{f} d t, \quad \omega^{i 7}=\frac{d \alpha_{1}}{d \rho} \sigma_{i}, \quad \bar{\omega}^{i 7}=\frac{d \alpha_{2}}{d \rho} \bar{\sigma}_{i}, \quad \tilde{\omega}^{i 7}=\frac{d \alpha_{1}}{d \rho} \tilde{\sigma}_{i}, \quad i=1,2, \\
& \omega^{12}=\sigma_{3}, \quad \omega^{\overline{1} \overline{2}}=\bar{\sigma}_{3}, \quad \omega^{\tilde{1} \tilde{2}}=\tilde{\sigma}_{3} .
\end{aligned}
$$

The split of the six-cycle into the product in (2.7) dictates the twist, and the only non-vanishing component of the gauge field is

$$
A^{3}=-\frac{1}{g}\left(\sigma_{3}+\bar{\sigma}_{3}+\tilde{\sigma}_{3}\right)
$$

where for simplicity the overall constant has already been set to the value consistent with supersymmetry. The $S U(2)_{R}$ symmetry of the unwrapped branes is therefore broken to $U(1)_{R}$. Geometrically, the breaking of the R-symmetry happens because there are two normal directions to the D6-branes that are inside the Calabi-Yau fourfold; the Rsymmetry is broken to the $U(1)_{R}$ on the 2-plane defined by them. The twist (2.12) amounts to the identification of this $U(1)_{R}$ with a $U(1)$ subgroup in one of the $S U(2)$ factors in the $S O(6)$ structure group of the six-cycle.

We will also introduce a four-form flux corresponding to D2-branes along the twosphere directions on the six-cycle. In order to keep democracy, we will turn on four-form components along all three two-spheres,

$$
\begin{aligned}
G_{x_{0} \rho \sigma_{1} \sigma_{2}} & =Q_{1} \frac{\alpha_{1}^{2}}{\alpha_{2}^{2} \alpha_{3}^{2}} e^{-2 \Phi+f}, \\
G_{x_{0} \rho \bar{\sigma}_{1} \bar{\sigma}_{2}} & =Q_{2} \frac{\alpha_{2}^{2}}{\alpha_{3}^{2} \alpha_{1}^{2}} e^{-2 \Phi+f}, \\
G_{x_{0} \rho \tilde{\sigma}_{1} \tilde{\sigma}_{2}} & =Q_{3} \frac{\alpha_{3}^{2}}{\alpha_{1}^{2} \alpha_{2}^{2}} e^{-2 \Phi+f} .
\end{aligned}
$$

where all directions above are curved, $Q_{1}, Q_{2}$ and $Q_{3}$ are dimensionfull constants and the specific functional dependence is uniquely fixed by the equation of motion for the three-form potential. Among the three two-form field strengths $G_{\mu \nu \alpha}$ we will choose as only non-vanishing the one corresponding to $\alpha=3$, thus complying with the requirements spelled out after (2.6). Then the field strength solving the equation of motion for the vector $B_{\mu 3}$ is

$$
G_{x_{0} \rho \alpha}=-Q_{4} \frac{e^{2 \Phi+f}}{\alpha_{1}^{2} \alpha_{2}^{2} \alpha_{3}^{2}} \delta_{\alpha 3}
$$


with $x_{0}$ again a curved direction and $Q_{4}$ dimensionfull. This ansatz points towards a particle associated with the one-form potential. However, this is only an artifact of the eight-dimensional description and in fact the flux (2.14) is due to D2-branes forming a bound state with the D6-branes. We should point out that turning on just the three-form and one-form potentials is not in general consistent with the full set of equations of motion. Since we have set to zero the scalar and the three two-form potentials (see footnote 1) the corresponding equations of motion constrain the remaining fields. ${ }^{2}$ For instance, if we set also $G_{\mu \nu \alpha}=0$, then the non-trivial constraints are

$$
\epsilon^{\mu_{1} \ldots \mu_{8}} G_{\mu_{1} \mu_{2} \mu_{3} \mu_{4}} G_{\mu_{5} \mu_{6} \mu_{7} \mu_{8}}=0, \quad G_{\mu \nu}{ }^{\rho \sigma} F_{\rho \sigma}^{\alpha}=0
$$

In our case, using (2.13) we find that (2.15) reduces to $Q_{1}+Q_{2}+Q_{3}=0$. When we turn on $G_{\mu \nu \alpha}$ as well, the generalization of (2.15) is quite complicated. However, for the case at hand with the flux components given by (2.13) and (2.14), we obtain the simple algebraic condition

$$
Q_{1}+Q_{2}+Q_{3}+Q_{4}=0
$$

namely, that the total flux charge vanishes.

Let's now turn to the scalars. Only the scalar corresponding to the unbroken $U(1)_{R}$ R-symmetry will survive the twisting, so that we will turn on one of the scalars $L_{\alpha}^{i}$,

$$
L_{\alpha}^{i}=\operatorname{diag}\left(e^{\lambda}, e^{\lambda}, e^{-2 \lambda}\right) .
$$

We will impose on the spinor the consistent projections

$$
\begin{aligned}
& \Gamma_{7} \epsilon=-i \Gamma_{9} \epsilon, \\
& \Gamma_{1} \Gamma_{2} \epsilon=\bar{\Gamma}_{1} \bar{\Gamma}_{2} \epsilon=\tilde{\Gamma}_{1} \tilde{\Gamma}_{2} \epsilon=-\hat{\Gamma}_{1} \hat{\Gamma}_{2} \epsilon .
\end{aligned}
$$

which leave in total two independent components for the spinor, so that at low energies we are left with a supersymmetric quantum mechanical model having two supercharges. The first projection effectively reduces the theory along the six-cycle and the other three project into singlet spinors of diagonal $U(1)$ 's. ${ }^{3}$

\footnotetext{
${ }^{2}$ We thank A. Paredes and A. V. Ramallo for a discussion on this point.

${ }^{3}$ A gravity dual for a quantum mechanics with two supercharges was also constructed in [19] using maximal gauged supergravity in seven dimensions to describe M-fivebranes wrapping a product of a three-cycle with a two-cycle.
} 
With the above ansatz and projections on the spinor, the supersymmetry variations for the gravitino and gaugino lead to the following equations

$$
\begin{aligned}
\frac{d \Phi}{d \rho} & =\frac{g}{8} e^{-\Phi}\left(e^{-4 \lambda}+2 e^{2 \lambda}\right)-\frac{1}{2 g} e^{\Phi-2 \lambda}\left(\frac{1}{\alpha_{1}^{2}}+\frac{1}{\alpha_{2}^{2}}+\frac{1}{\alpha_{3}^{2}}\right) \\
& -\frac{\left(\alpha_{1}^{2} Q_{1}+\alpha_{2}^{2} Q_{2}+\alpha_{3}^{2} Q_{3}\right) e^{-\Phi}-Q_{4} e^{\Phi+2 \lambda}}{2 \alpha_{1}^{2} \alpha_{2}^{2} \alpha_{3}^{2}}, \\
\frac{1}{\alpha_{1}} \frac{d \alpha_{1}}{d \rho} & =\frac{g}{24} e^{-\Phi}\left(2 e^{2 \lambda}+e^{-4 \lambda}\right)+\frac{1}{6 g} e^{\Phi-2 \lambda}\left(\frac{5}{\alpha_{1}^{2}}-\frac{1}{\alpha_{2}^{2}}-\frac{1}{\alpha_{3}^{2}}\right) \\
& -\frac{\left(-\alpha_{1}^{2} Q_{1}+\alpha_{2}^{2} Q_{2}+\alpha_{3}^{2} Q_{3}\right) e^{-\Phi}+\frac{1}{3} Q_{4} e^{\Phi+2 \lambda}}{2 \alpha_{1}^{2} \alpha_{2}^{2} \alpha_{3}^{2}}, \\
\frac{1}{\alpha_{2}} \frac{d \alpha_{2}}{d \rho} & =\frac{g}{24} e^{-\Phi}\left(2 e^{2 \lambda}+e^{-4 \lambda}\right)+\frac{1}{6 g} e^{\Phi-2 \lambda}\left(\frac{5}{\alpha_{2}^{2}}-\frac{1}{\alpha_{3}^{2}}-\frac{1}{\alpha_{1}^{2}}\right) \\
& -\frac{\left(\alpha_{1}^{2} Q_{1}-\alpha_{2}^{2} Q_{2}+\alpha_{3}^{2} Q_{3}\right) e^{-\Phi}+\frac{1}{3} Q_{4} e^{\Phi+2 \lambda}}{2 \alpha_{1}^{2} \alpha_{2}^{2} \alpha_{3}^{2}}, \\
\frac{1}{\alpha_{3}} \frac{d \alpha_{3}}{d \rho} & =\frac{g}{24} e^{-\Phi}\left(2 e^{2 \lambda}+e^{-4 \lambda}\right)+\frac{1}{6 g} e^{\Phi-2 \lambda}\left(\frac{5}{\alpha_{3}^{2}}-\frac{1}{\alpha_{1}^{2}}-\frac{1}{\alpha_{2}^{2}}\right) \\
& -\frac{\left(\alpha_{1}^{2} Q_{1}+\alpha_{2}^{2} Q_{2}-\alpha_{3}^{2} Q_{3}\right) e^{-\Phi}+\frac{1}{3} Q_{4} e^{\Phi+2 \lambda}}{2 \alpha_{1}^{2} \alpha_{2}^{2} \alpha_{3}^{2}}, \\
\frac{d \lambda}{d \rho} & =\frac{g}{6} e^{-\Phi}\left(e^{-4 \lambda}-e^{2 \lambda}\right)+\frac{1}{3 g} e^{\Phi-2 \lambda}\left(\frac{1}{\alpha_{1}^{2}}+\frac{1}{\alpha_{2}^{2}}+\frac{1}{\alpha_{3}^{2}}\right)+\frac{Q_{4} e^{\Phi+2 \lambda}}{3 \alpha_{1}^{2} \alpha_{2}^{2} \alpha_{3}^{2}}, \\
\frac{d f}{d \rho} & =\frac{1}{3} \frac{d \Phi}{d \rho}+\frac{2\left(\alpha_{1}^{2} Q_{1}+\alpha_{2}^{2} Q_{2}+\alpha_{3}^{2} Q_{3}\right) e^{-\Phi}+2 Q_{4} e^{\Phi+2 \lambda}}{3 \alpha_{1}^{2} \alpha_{2}^{2} \alpha_{3}^{2}} .
\end{aligned}
$$

Furthermore, we also obtain from the gravitino variation along $\rho$ a differential equation yielding the $\rho$-dependence of the Killing spinor as $\epsilon=e^{f / 2} \epsilon_{0}$, where $\epsilon_{0}$ is a constant spinor subject to the projections (2.18). In fact, this functional form of the Killing spinor can be deduced just from the supersymmetry algebra.

We also note that the system (2.19) also includes the case when the six-cycle is taken to be $S^{2} \times \mathbb{C P}^{2}$; this corresponds to setting equal radii for two of the spheres. We only have to adjust the overall scale in the metric for $\mathbb{C P}^{2}$ to have scalar curvature equal to that of the metric for the undeformed $S^{2} \times S^{2}$, which, in our normalization, equals four.

In order to solve the system (2.19) it is useful to redefine variables through

$$
\begin{aligned}
& d r=e^{-\Phi / 3} d \rho, \quad a_{i}=\alpha_{i} e^{-\Phi / 3}, \quad i=1,2,3, \\
& a_{4}=e^{\lambda+2 \Phi / 3}, \quad a=e^{-2 \lambda+2 \Phi / 3}, \quad A=f-\frac{\Phi}{3} \text {. }
\end{aligned}
$$


In these variables the metric, when lifted to eleven dimensions, takes the form (in what follows we have conveniently set $g=2$; it can be reinstalled or taken to any value after appropriate rescalings)

$$
d s_{11}^{2}=-e^{2 A} d t^{2}+d r^{2}+a_{1}^{2} d \Omega_{2}^{2}+a_{2}^{2} d \bar{\Omega}_{2}^{2}+a_{3}^{2} d \tilde{\Omega}_{2}^{2}+a_{4}^{2} d \hat{\Omega}_{2}^{2}+a^{2}\left(\hat{\sigma}_{3}-\sigma_{3}-\bar{\sigma}_{3}-\tilde{\sigma}_{3}\right)^{2}
$$

where the $\hat{\sigma}_{i}$ 's are left-invariant Maurer-Cartan $S U(2)$ one-forms satisfying as a triplet the conditions $d \hat{\sigma}_{i}=\frac{1}{2} \epsilon_{i j k} \hat{\sigma}_{j} \wedge \hat{\sigma}_{k}$. The Killing spinor can also be lifted from eight to eleven dimensions through $\epsilon_{11}=e^{-\Phi / 6} \epsilon=e^{A / 2} \epsilon_{0}$, while the eleven-dimensional four-form field strength corresponding to the uplift of (2.13) is given by

$$
\begin{array}{ll}
F_{0712}=\frac{Q_{1}}{a_{2}^{2} a_{3}^{2} a_{4}^{2} a}, & F_{07 \overline{1} \overline{2}}=\frac{Q_{2}}{a_{1}^{2} a_{3}^{2} a_{4}^{2} a}, \\
F_{07 \tilde{1} \tilde{2}}=\frac{Q_{3}}{a_{1}^{2} a_{2}^{2} a_{4}^{2} a}, & F_{07 \hat{1} \hat{2}}=\frac{Q_{4}}{a_{1}^{2} a_{2}^{2} a_{3}^{2} a} .
\end{array}
$$

After the redefinitions (2.20), the system (2.19) becomes simpler,

$$
a_{1} \frac{d a_{1}}{d r}=\frac{a}{2}-\frac{1}{3} \frac{1}{a_{1}^{2} a_{2}^{2} a_{3}^{2} a_{4}^{2} a}\left(-2 a_{1}^{2} Q_{1}+a_{2}^{2} Q_{2}+a_{3}^{2} Q_{3}+a_{4}^{2} Q_{4}\right)
$$

plus three more equations following from cyclic permutations in 1,2,3,4, as well as

$$
\frac{d a}{d r}=1-\frac{a^{2}}{2}\left(\frac{1}{a_{1}^{2}}+\frac{1}{a_{2}^{2}}+\frac{1}{a_{3}^{2}}+\frac{1}{a_{4}^{2}}\right)-\frac{1}{3} \frac{1}{a_{1}^{2} a_{2}^{2} a_{3}^{2} a_{4}^{2}}\left(a_{1}^{2} Q_{1}+a_{2}^{2} Q_{2}+a_{3}^{2} Q_{3}+a_{4}^{2} Q_{4}\right)
$$

whose solution determines the conformal factor as

$$
\frac{d A}{d r}=\frac{2}{3} \frac{1}{a_{1}^{2} a_{2}^{2} a_{3}^{2} a_{4}^{2} a}\left(a_{1}^{2} Q_{1}+a_{2}^{2} Q_{2}+a_{3}^{2} Q_{3}+a_{4}^{2} Q_{4}\right)
$$

The above system should be supplemeted by the zero total charge condition (2.16) and obvisously shares an $S_{4}$ permutation invariance originating from the equivalence of all four two-spheres in the background (2.21) and (2.22).

We also note that having unequal radii for the spheres in the six-cycle provides the possibility to perform the limit where the radius of one of the two-spheres tends to infinity. This would amount to the growth of two flat coordinates; for instance for very large $a_{4}$ it is easily seen that $2+1$ Lorentz invariance is restored and the system (2.23)-(2.25) (and the equivalent in (2.19)) becomes that in [12]. In the absence of flux the metric (2.21) becomes $d s_{1,2}^{2}+d s_{8}^{2}$, where $d s_{8}^{2}$ is the metric of a Calabi-Yau fourfold, thus reducing from $S U(5)$ to $S U(4)$ holonomy. Similarly, one can go from $S U(4)$ to $S U(3)$ holonomy by blowing up one of the remaining two-spheres. 
Turning on fluxes as in (2.22) corresponds, from a string theory point of view, to turning on D2-brane charges and forming a D2-D6 bound state with the entire spatial part of the world-volume wrapped on the spheres. This interpretation is compatible with the fact that no additional projection is required as compared with the zero flux case and the amount of supersymmetry preserved is the same. We have been unable to solve the system (2.24)(2.25), unlike the similar case in [12], in the presence of fluxes. In that respect note that having non-vanishing fluxes is inconsistent with demanding simple solutions with equal radii, i.e. $a_{1}=\cdots=a_{4}$.

In subsection 3.1 we will show how to derive (2.23)-(2.25) with (2.16) directly using eleven-dimensional supergravity.

\section{$2.1 \quad S U(5)$ holonomy}

Consider the case with vanishing fluxes, $Q_{i}=0$, with $i=1,2,3,4$. In this case the general solution to the system (2.23)-(2.24) is (the conformal factor equals unity in this case)

$$
a_{1}^{2}=R^{2}+l_{1}^{2}, \quad a_{2}^{2}=R^{2}+l_{2}^{2}, \quad a_{3}^{2}=R^{2}+l_{3}^{2}, \quad a_{4}^{2}=R^{2}, \quad a^{2}=R^{2} U^{2}(R)
$$

where

$$
U^{2}(R)=\frac{12 R^{6}+15 C_{1} R^{4}+20 C_{2} R^{2}+30 C_{3}+12 C / R^{4}}{30\left(R^{2}+l_{1}^{2}\right)\left(R^{2}+l_{2}^{2}\right)\left(R^{2}+l_{3}^{2}\right)},
$$

with the constants $C_{i}, i=1,2,3$ expressed as symmetric homogeneous polynomials, up to cubic order, in the $l_{i}^{2}$ 's

$$
C_{1}=l_{1}^{2}+l_{2}^{2}+l_{3}^{2}, \quad C_{2}=l_{1}^{2} l_{2}^{2}+l_{2}^{2} l_{3}^{2}+l_{3}^{2} l_{1}^{2}, \quad C_{3}=l_{1}^{2} l_{2}^{2} l_{3}^{2}
$$

The relation of the two variables $r$ and $R$ is via the differential

$$
d r=\frac{2}{U(R)} d R
$$

Here we have denoted four of the constants of integration by $l_{1}, l_{2}, l_{3}$ and $C$, and we have absorbed the fifth one by an appropriate shift in the variable $R{ }^{4}$ We can also see that in this case $e^{2 \Phi}=R^{3} U(R), f=\Phi / 3$ and $A=0$. Hence the lifted eleven-dimensional solution in (2.21) factorizes into the time coordinate and a Calabi-Yau fivefold with metric

$$
d s_{10}^{2}=d r^{2}+a_{1}^{2} d \Omega_{2}^{2}+a_{2}^{2} d \bar{\Omega}_{2}^{2}+a_{3}^{2} d \tilde{\Omega}_{2}^{2}+a_{4}^{2} d \hat{\Omega}_{2}^{2}+a^{2}\left(\hat{\sigma}_{3}-\sigma_{3}-\bar{\sigma}_{3}-\tilde{\sigma}_{3}\right)^{2} .
$$

\footnotetext{
${ }^{4}$ The symmetry between all two-spheres can be manifestly restored in the solution (2.26) if we make the variable shift $R^{2} \rightarrow R^{2}+l_{4}^{2}$ and simultaneously redefine $l_{i}^{2} \rightarrow l_{i}^{2}-l_{4}^{2}$ for $i=1,2,3$.
} 
The asymptotic behaviour for large values of $R$ takes the universal form

$$
d s_{10}^{2} \simeq 10 d R^{2}+R^{2} d s_{9}^{2}, \quad \text { as } \quad R \rightarrow \infty
$$

and it describes a cone whose base is given by the nine-dimensional metric

$$
d s_{9}^{2}=d \Omega_{2}^{2}+d \bar{\Omega}_{2}^{2}+d \tilde{\Omega}_{2}^{2}+d \hat{\Omega}_{2}^{2}+\frac{2}{5}\left(\hat{\sigma}_{3}-\sigma_{3}-\bar{\sigma}_{3}-\tilde{\sigma}_{3}\right)^{2} .
$$

This is an Einstein space obeying $R_{i j}=\frac{4}{5} g_{i j}$. In fact it is the symmetric coset space

$$
\frac{S U(2) \times S U(2) \times S U(2) \times S U(2)}{U(1) \times U(1) \times U(1)},
$$

where the embedding of the $U(1)$ 's into the Cartan subalgebra in the numerator is diagonal. We have been unable to find a previous reference and a nomenclature for it in the literature, but this is simply a higher dimensional analog of the largest similar case appearing as a Freund-Rubin compactification [20] of eleven-dimensional supergravity [18] in four dimensions, i.e. $Q^{1,1,1}[21]$. Extending that nomenclature we will refer to this space as $\Pi^{1,1,1,1}$.

In fact, (2.32) is an exact solution for all values of $R$ as it can also be obtained by letting $l_{1}=l_{2}=l_{3}=C=0$ in the general solution. However, extending (2.32) to the interior is problematic because we reach a singularity at $R=0$, where the fiber and the $S^{2}$ 's collapse to a point. Resolving the singularity to avoid this collapse requires that we turn on some of the different moduli parameters which also determine the behavior of the solution in the interior. In the following we further analyze for two different cases the solution for generic ranges of the parameters and show that indeed the singularity can be resolved in a manner similar to that in [12].

$\underline{l_{1}=l_{2}=l_{3}=0}$ : In this case, when the constant $C \geq 0$, the variable $R \geq 0$ and then the manifold is singular at $R=0$. If, however, $C=-\rho_{0}^{10}<0$, where $\rho_{0}$ is a real positive constant, then the variable $R \geq \rho_{0}$ and the metric takes the simple form

$$
\begin{aligned}
d s_{10}^{2}= & \frac{10 d R^{2}}{1-\rho_{0}^{10} / R^{10}}+R^{2}\left(d \Omega_{2}^{2}+d \bar{\Omega}_{2}^{2}+d \tilde{\Omega}_{2}^{2}+d \hat{\Omega}_{2}^{2}\right) \\
& +\frac{2}{5} R^{2}\left(1-\rho_{0}^{10} / R^{10}\right)\left(\hat{\sigma}_{3}-\bar{\sigma}_{3}-\tilde{\sigma}_{3}-\hat{\sigma}_{3}\right)^{2} .
\end{aligned}
$$

Near $R=\rho_{0}$ we change to a new radial variable $\tau=2 \sqrt{\rho_{0}\left(R-\rho_{0}\right)}$ and find the behavior

$$
d s_{10}^{2} \simeq a^{2}\left(d \Omega_{2}^{2}+d \bar{\Omega}_{2}^{2}+d \tilde{\Omega}_{2}^{2}+d \hat{\Omega}_{2}^{2}\right)+d \tau^{2}+\tau^{2}\left(\hat{\sigma}_{3}-\sigma_{3}-\bar{\sigma}_{3}-\tilde{\sigma}_{3}\right)^{2}, \text { as } \tau \rightarrow 0 .
$$


Therefore, near $\tau=0$ (or equivalently $R=\rho_{0}$ ) and for constant $\theta$ and $\phi$, as well as for the corresponding barred, tilded and hatted angles, the metric behaves as $d t^{2}+t^{2} d \hat{\psi}^{2}$ which shows that $t=0$ is a bolt singularity [22] which is removable provided that the periodicity

of the angle $\hat{\psi}$ is restricted to $0 \leq \hat{\psi}<2 \pi$. Then the space becomes topologically $S^{2} \times$ $S^{2} \times S^{2} \times S^{2} \times \mathbb{R}^{2}$ and the full solution interpolates between this space for $R \rightarrow a$ and the ten-dimensional space (2.31) for $R \rightarrow \infty$. However, the latter is now a cone with base $\Pi^{1,1,1,1} / \mathbb{Z}_{2}$ due to the above discrete identification.

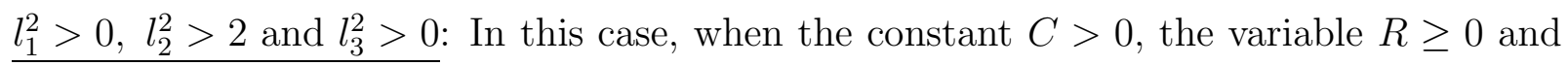
there is a singularity at $R=0$. If, however, $C=0$ then we have the behavior

$$
\begin{aligned}
d s_{10}^{2} \simeq & l_{1}^{2} d \Omega_{2}^{2}+l_{2}^{2} d \bar{\Omega}_{2}^{2}+l_{3}^{2} d \tilde{\Omega}_{2}^{2}+4 d R^{2}+R^{2} d \hat{\Omega}_{2}^{2}+R^{2}\left(\hat{\sigma}_{3}-\sigma_{3}-\bar{\sigma}_{3}-\tilde{\sigma}_{3}\right)^{2} \\
& \text { as } \quad R \rightarrow 0 .
\end{aligned}
$$

Hence, for constant $\theta, \phi, \bar{\theta}, \bar{\phi}$ and $\tilde{\theta}, \tilde{\phi}$ the metric behaves as $4 d R^{2}+R^{2}\left(\hat{\sigma}_{1}^{2}+\hat{\sigma}_{2}^{2}+\hat{\sigma}_{3}^{2}\right)$ which shows that we simply have a coordinate singularity in the polar coordinate system on an $\mathbb{R}^{4}$ centered at $R=0$. This is the so called nut singularity [22], which is removable by adding the point $R=0$ and changing to Cartesian coordinates. Therefore near $R=0$ the manifold becomes topologically $S^{2} \times S^{2} \times S^{2} \times \mathbb{R}^{4}$. Then the full solution interpolates between this space for $R \rightarrow 0$ and the ten-dimensional flat space in (2.31) for $R \rightarrow \infty$. If $C<0$ then there is an $R_{0}$ such that $U\left(R_{0}\right)^{2}=0$ (we take the largest root of this sixth order, in $R_{0}^{2}$, algebraic equation) and therefore we have that $R \geq R_{0}$. Changing to a new radial variable $\tau=2 \sqrt{R_{0}\left(R-R_{0}\right)}$ we find the behavior

$$
\begin{aligned}
d s_{10}^{2} \simeq & \left(R_{0}^{2}+l_{1}^{2}\right) d \Omega_{2}^{2}+\left(R_{0}^{2}+l_{2}^{2}\right) d \bar{\Omega}_{2}^{2}+\left(R_{0}^{2}+l_{3}^{2}\right) d \tilde{\Omega}_{2}^{2}+R_{0}^{2} d \hat{\Omega}_{2}^{2} \\
& +d \tau^{2}+\tau^{2}\left(\hat{\sigma}_{3}-\sigma_{3}-\bar{\sigma}_{3}\right)^{2}, \quad \text { as } \quad \tau \rightarrow 0 .
\end{aligned}
$$

Hence the behavior is similar to that found before in (2.35), with a removable bolt singularity at $\tau=0$.

\section{$3 \quad S U(n+1)$ holonomy}

The case of D6-branes wrapped on six-cycles with $S^{2} \times \bar{S}^{2} \times \tilde{S}^{2}$ topology we have considered in this paper is a generalization of that of D6-branes wrapped on $S^{2} \times \bar{S}^{2}$ four-cycles, leading to $S U(4)$ holonomy [7, 12], or $S^{2}$ two-cycles corresponding to the resolved [5] or 
deformed conifold [6]. These constructions can be extended to an arbitrary even number of dimensions $2 n+2$ and $S U(n+1)$ holonomy. Of course for $n \geq 5$ we give up the brane description of the underlying geometry. Consider the metric

$$
d s_{2 n+2}^{2}=d r^{2}+\sum_{i=1}^{n} a_{i}^{2} d \Omega_{2, i}^{2}+a^{2}\left(d \psi+\sum_{i=1}^{n} \sigma_{3, i}\right)^{2}
$$

containing a set of $n$ spheres, with $d \Omega_{2, i}^{2}=\sigma_{1, i}^{2}+\sigma_{2, i}^{2}$ and where the relation $d \sigma_{3, i}=\sigma_{1, i} \wedge \sigma_{2, i}$ defines the $\sigma_{3, i}$ 's.

In the natural frame

$$
e_{i}^{1}=a_{i} \sigma_{1, i}, \quad e_{i}^{2}=a_{i} \sigma_{2, i}, \quad e^{2 n+1}=a\left(d \psi+\sum_{i=1}^{n} \sigma_{3, i}\right), \quad e^{2 n+2}=d r
$$

the spin connection in a quite obvious notation reads

$$
\begin{aligned}
& \omega_{(i)}^{12}=\sigma_{3, i}-\frac{a^{2}}{2 a_{i}^{2}}\left(d \psi+\sum_{i=1}^{n} \sigma_{3, i}\right), \quad \omega^{2 n+12 n+2}=\frac{d a}{d r}\left(d \psi+\sum_{i=1}^{n} \sigma_{3, i}\right) \\
& \omega_{(i)}^{12 n+2}=\frac{d a_{i}}{d r} \sigma_{1, i}, \quad \omega_{(i)}^{22 n+2}=\frac{d a_{i}}{d r} \sigma_{2, i}, \quad \omega_{(i)}^{2 n+11}=\frac{a}{2 a_{i}} \sigma_{2, i}, \quad \omega_{(i)}^{22 n+1}=\frac{a}{2 a_{i}} \sigma_{1, i} .
\end{aligned}
$$

In order to generalize our construction to arbitrary even dimensions we will first extend the projections on the spinor as

$$
\Gamma_{(1)}^{1} \Gamma_{(1)}^{2} \epsilon=\ldots=\Gamma_{(n)}^{1} \Gamma_{(n)}^{2} \epsilon=-\Gamma^{2 n+1} \Gamma^{2 n+2} \epsilon
$$

representing, in total, $2^{n}$ independent conditions. The metric (3.1) will admit a covariantly constant spinor provided that the first order system of differential equations ${ }^{5}$

$$
\begin{aligned}
a_{i} \frac{d a_{i}}{d r} & =\frac{a}{2}, \quad i=1, \ldots, n \\
\frac{d a}{d r} & =1-\frac{a^{2}}{2} \sum_{i=1}^{n} \frac{1}{a_{i}^{2}},
\end{aligned}
$$

is obeyed. The form of the covariantly constant spinor turns out to be $\epsilon=e^{\Gamma_{(1)}^{12} \psi / 2} \epsilon_{0}$, with $\epsilon_{0}$ a constant spinor subject to the same projections as in (3.4). These projections leave two independent components for the spinor, so that we are left with an $S U(n+1)$ holonomy metric.

\footnotetext{
${ }^{5}$ This system was also studied in [23].
} 
The general solution to the system $(3.5)$ is

$$
\begin{aligned}
& d r=\frac{2}{U(R)} d R, \quad a^{2}=R^{2} U^{2}(R), \\
& a_{i}^{2}=R^{2}+l_{i}^{2}, \quad \text { with } i=1, \ldots, n \text { and } l_{n}^{2}=0,
\end{aligned}
$$

where

$$
U^{2}(R)=\frac{2}{\prod_{i=1}^{n-1}\left(R^{2}+l_{i}^{2}\right)}\left[\frac{C / R^{4}}{n+1}+\sum_{i=0}^{n-1} \frac{R^{2 i}}{i+2} S_{n-i-1}\left(l^{2}\right)\right],
$$

with $C$ being a constant and $S_{m}\left(l^{2}\right)$ a symmetric homogeneous polynomial in the $l_{i}^{2}$ 's, of degree $m$, with $m=0,1, \ldots, n-1$,

$$
S_{m}\left(l^{2}\right)=\sum_{i_{1}<\ldots<i_{m}=1}^{n-1} l_{i_{1}}^{2} l_{i_{2}}^{2} \ldots l_{i_{m}}^{2} .
$$

The asymptotic behaviour of (3.1) as $R \rightarrow \infty$ is that of a cone with metric

$$
d s_{2 n+2}^{2}=2(n+1) d R^{2}+R^{2} d s_{2 n+1}^{2}
$$

and base the $(2 n+1)$-dimensional Einstein space

$$
d s_{2 n+1}^{2}=\sum_{i=1}^{n} d \Omega_{2, i}^{2}+\frac{2}{n+1}\left(d \psi+\sum_{i=1}^{n} \sigma_{3, i}\right)^{2},
$$

with $R_{i j}=\frac{n}{n+1} g_{i j}$. This is the symmetric coset space

$$
\frac{\overbrace{S U(2) \times S U(2) \times \ldots \times S U(2)}^{n \text { factors }}}{\underbrace{U(1) \times \ldots \times U(1)}_{n-1 \text { factors }}},
$$

and we can denote it, by extending our previous notation corresponding to $n=4$, as $\Pi^{1,1, \ldots, 1}$, with $n$ indices. In the case where all $l_{i}=0$ and $C=-\rho_{0}^{2 n+2}<0$ we have

$$
U^{2}(R)=\frac{2}{n+1}\left(1-\frac{\rho_{0}^{2 n+2}}{R^{2 n+2}}\right) .
$$

Therefore near $R=\rho_{0}$ we find a removable bolt singularity with $S_{(1)}^{2} \times S_{(2)}^{2} \times \ldots S_{(n)}^{2} \times \mathbb{R}^{2}$ topology. When $C=0$, and all $l_{i}^{2}>0$, a removable nut singularity arises near $R=0$, with $S_{(1)}^{2} \times S_{(2)}^{2} \times \ldots S_{(n-1)}^{2} \times \mathbb{R}^{4}$ topology.

Obviously the cases with $n \geq 5$ do not have an interpretation in terms of branes and therefore seem to be of no direct interest to string and M-theory. Nevertheless, we find it quite interesting that the general structure is extendable for any $n$ and we believe that the explicit forms of the metrics we have presented will be useful in general. 


\subsection{Non-vanishing flux from eleven-dimensional supergravity}

It is worth examining the case of non-vanishing flux with background given by (2.21) and (2.22) directly using eleven-dimensional supergravity. In particular, it is worth seeing how the condition (2.16) arises in this approach. The Killing spinor equation is

$$
\partial_{\mu} \epsilon+\frac{1}{4} \omega_{\mu}^{a b} \Gamma_{a b} \epsilon-\frac{1}{288}\left(F_{\nu \rho \lambda \sigma} \Gamma_{\mu}^{\nu \rho \lambda \sigma}-8 F_{\mu \nu \rho \lambda} \Gamma^{\nu \rho \lambda}\right) \epsilon=0 .
$$

The non-zero components of the spin connection are given, after appropriate relabeling, by (3.3), where the general case should be specialized to $n=4$ and supplemented with $\omega^{0,10}=$ $e^{A} d A / d r d t$. Similarly, the appropriate projections are given by (3.4) (with $n=4$ ). It turns out that the Killing spinor is $\epsilon=e^{A / 2} e^{\frac{1}{2} \Gamma_{12} \psi} \epsilon_{0}$, provided that the system of equations (2.23)-(2.25) is obeyed (with charges rescaled by the factor -2 ), with no restriction, such as (2.16), for the charges. However, we have to make sure that the equation of motion for the three-form gauge potential is obeyed. For the ansatz (2.22) the contribution to it from the Chern-Simons term in the action vanishes. Hence, we have that

$$
\frac{1}{\sqrt{-g}} \partial_{\mu}\left(\sqrt{-g} F^{\mu \nu \rho \lambda}\right)=0 .
$$

For every choice of $\nu, \rho$ and $\lambda$ this is trivially satisfied with the background ansatz (2.21) and (2.22). However, satisfying the equation for $(\nu, \rho, \lambda)=(t, r, \psi)$ requires imposing the zero total charge condition (2.16).

\section{Acknowledgments}

R.H. acknowledges the financial support provided through the European Community's Human Potential Programme under contract HPRN-CT-2000-00131 "Quantum Structure of Space-time", the Swiss Office for Education and Science and the Swiss National Science Foundation.

K.S. acknowledges the financial support provided through the European Community's Human Potential Programme under contracts HPRN-CT-2000-00131 "Quantum Structure of Space-time" and HPRN-CT-2000-00122 "Superstring Theory", by the Greek State Scholarships Foundation under the contract IKYDA-2001/22 "Quantum Fields and Strings", as well as NATO support by a Collaborative Linkage Grant under the contract PST.CLG.978785 "Algebraic and Geometrical Aspects of Conformal Field Theories and Superstrings". He also acknowledges the hospitality and financial support of the THDivision of CERN, where a substantial part of this work was done. 


\section{References}

[1] J. Gomis, Nucl. Phys. B606 (2001) 3, hep-th/0103115.

[2] M. Bershadsky, C. Vafa and V. Sadov, Nucl. Phys. B463 (1996) 420, hep-th/9511222.

[3] J. Maldacena and C. Núñez, Int. J. Mod. Phys. A16 (2001) 822, hep-th/0007018 and Phys. Rev. Lett. 86 (2001) 588, hep-th/0008001.

[4] J. P. Gauntlett, Branes, calibrations and supergravity, hep-th/0305074.

[5] J.D. Edelstein and C. Núñez, JHEP 0104 (2001) 028, hep-th/0103167.

[6] J. D. Edelstein, A. Paredes and A. V. Ramallo, Phys. Lett. B 554 (2003) 197, hep-th/0212139.

[7] J. Gomis and T. Mateos, Phys. Lett. B524 (2002) 170, hep-th/0108080.

[8] R. Hernández and K. Sfetsos, Phys. Lett. B536 (2002) 294, hep-th/0202135.

[9] J. D. Edelstein, A. Paredes and A. V. Ramallo, JHEP 0301 (2003) 011, hep-th/0211203.

[10] R. Hernández, Phys. Lett. B521 (2001) 371, hep-th/0106055.

[11] U. Gursoy, C. Núñez and M. Schvellinger, JHEP 0206 (2002) 015, hep-th/0203124.

[12] R. Hernández and K. Sfetsos, JHEP 0207 (2002) 045, hep-th/0205099.

[13] J. Brugués, J. Gomis, T. Mateos and T. Ramírez, JHEP 0210 (2002) 016, hep-th/0207091.

[14] J. D. Edelstein, A. Paredes and A. V. Ramallo, JHEP 0212 (2002) 075, hep-th/0207127.

[15] J. Brugués, J. Gomis, T. Mateos and T. Ramírez, Class. Quant. Grav. 20 (2003) S441, hep-th/0212179.

[16] R. Hernández and K. Sfetsos, Class. Quant. Grav. 20 (2003) S501, hep-th/0211130.

[17] A. Salam and E. Sezgin, Nucl. Phys. B258 (1985), 284.

[18] E. Cremmer, B. Julia and J. Scherk, Phys. Lett. B76 (1978) 409;

E. Cremmer and B. Julia, Nucl. Phys. B159 (1979) 141.

[19] J. P. Gauntlett and N. Kim, Phys. Rev. D65 086003 (2002), hep-th/0109039.

[20] P.G. Freund and M.A. Rubin, Phys. Lett. B97 (1980) 233.

[21] R. D'Auria, P. Fre and P. van Nieuwenhuizen, Phys. Lett. B136 (1984) 347.

[22] G. W. Gibbons and S. W. Hawking, Commun. Math. Phys. 66 (1979) 291;

T. Eguchi and A.J. Hanson, Annals Phys. 120 (1979) 82.

[23] M. Cvetic, G. W. Gibbons, H. Lu and C. N. Pope, Commun. Math. Phys. 232 (2003) 457, hep-th/0012011. 